\title{
Existence and Structure of Stochastic Equilibria with Intertemporal Substitution ${ }^{\dagger}$
}

\author{
By Peter Bank ANd Frank RiedeL ${ }^{\ddagger}$ \\ Humboldt University of Berlin \\ February, 2000 - this Version: December 13, 2000
}

\begin{abstract}
We prove existence of an Arrow-Debreu equilibrium when agents' preferences exhibit local substitution in the sense of Hindy, Huang, and Kreps (1992). Efficient allocations and supporting price functionals are identified and characterized. Under Hindy-Huang-Kreps preferences, equilibrium price processes are semimartingales for arbitrary endowments, but in general, they do not belong to the space suggested originally by Hindy and Huang (1992) due to an additional singular local time component.
\end{abstract}

\footnotetext{
† Support of Deutsche Forschungsgemeinschaft through SFB 373, "Quantification and Simulation of Economic Processes", is gratefully acknowledged.

$\ddagger$ We would like to thank Darrel Duffie for suggesting a more general approach, and Ulrich Horst for helpful discussions.

AMS 2000 subject classification. Primary 91B50; secondary 91B70

JEL subject classification. D51, D91

Key words and phrases. General Equilibrium, Local Substitution, Non-Time Additive Utility
} 


\section{Introduction}

Consumption of a good at one time is a close substitute for consumption of the same good an instant later. Therefore, utility functionals for intertemporal consumption plans should react in a continuous way when a consumption plan is shifted slightly over time. Now, the standard intertemporal utility functional widely used in economics and finance does not have this desirable property as has been shown by Hindy, Huang, and Kreps (1992). This gives an incentive to build up a new theory of intertemporal choice with preferences having this desirable continuity property. One part of this program is to develop general equilibrium theory for such preferences. This is the aim of the present paper.

Concerning our setup, we follow in spirit if not literally the fundamental work of Hindy, Huang, and Kreps (1992). Consumption patterns are described in cumulative form, i.e., as rightcontinuous, increasing processes on $[0, T]$. Of course, these patterns also have to be adapted to the information flow in the economy. To capture the substitutability of consumption over time, we endow the consumption space with the topology of weak convergence in probability plus $L^{1}(\mathbb{P})$-convergence of total masses. Agents are characterized by utility functionals which are continuous in this topology. HindyHuang-Kreps utilities are a possible choice.

For such an economy, we prove existence of a general equilibrium and analyze the structure of equilibrium prices.

As usual in the context of infinite dimensional commodity spaces, the Negishimethod is the basis for the proof of existence. In contrast to the usual approach as, e.g., in Mas-Colell and Richard (1991), however, we do not restrict price functionals a priori to be continuous on the consumption space. This continuity is established only a posteriori under the additional assumption that the information flow in the economy is quasi-leftcontinuous and that utility gradients are semimartingales with a continuous compensator. The general structure of supporting prices reveals that, without this extra structural requirements, there may actually be no continuous equilibrium price functionals for our economy.

If utility gradients are semimartingales, so are equilibrium prices as they take the form of a weighted maximum of such gradients. This is notable since semimartingale prices can be ensured in the time-additive model only by assuming that the aggregate endowment rate follows a semimartingale. Here, price processes have this particularly nice structure for any kind of endowment stream.

In contrast to a conjecture in Hindy and Huang (1992), however, it is not possible to ensure existence of an interest rate in general. Technically, this follows from an application of the Ito-Tanaka formula to the mentioned maximum representation of equilibrium prices. It turns out that the predictable part of equilibrium prices typically has a singular local time component, even when the basic utility gradients are compensated by absolutely continuous processes. The economic reason for this singularity is that agents refrain from consuming totally when prices are too high as compared to 
their marginal utility of consumption, and only resume consumption after a decline of prices. Hence, it may happen that the identity of the agent whose gradient sets the price changes when one agent stops consuming and another one restarts. If prices fluctuate in a diffusion-like manner, such periods of changes may be arbitrarily small, leading to a singular component in the evolution of equilibrium prices. Essentially the same singularity effect can also be observed in time-additive settings when marginal utility at zero is finite as has been shown by Karatzas, Lehoczky, and Shreve (1991).

Our approach to prove existence of equilibria relies on a Kuhn-Tucker like characterization of efficient allocations and on the structure of supporting prices as weighted maxima of utility gradients. A central technical tool is Komlós' theorem (Komlós (1967)). In its version by Kabanov (1999), this result gives us a powerful compactness principle which we use to prove both existence of efficient allocations and their continuous dependence on agents' weights. In conjunction with an argument going back to Bewley (1969), this continuity allows us to prove upper-hemicontinuity of the usual excess utility correspondence. Existence of equilibrium is then obtained by applying Kakutani's fixed point theorem.

The paper is organized as follows. Section 1 describes the economy under uncertainty, the consumption space and agents' utility functionals. Section 2 analyzes efficient allocations and their supporting prices. Section 3 establishes existence of general equilibrium for this economy. Finally, the continuity properties of equilibrium price functionals are studied in Section 4.

\section{The Economy}

We consider a stochastic pure exchange economy with a finite set of agents $\mathcal{I}$. Uncertainty is modeled through a filtered probability space $\left(\Omega, \mathcal{F}_{T},\left(\mathcal{F}_{t}, 0 \leq t \leq T\right), \mathbb{P}\right)$ satisfying the usual conditions of right continuity and completeness; $\mathcal{F}_{0}$ is $\mathbb{P}$-a.s. trivial.

\subsection{Consumption Plans and Price Functionals}

Under certainty, cumulative consumption is represented by an increasing, rightcontinuous function in

$$
\mathcal{M}_{+} \triangleq\{F:[0, T] \rightarrow[0,+\infty) \mid F \text { is rightcontinuous and increasing }\} .
$$

Since small shifts over time affect an agent's appreciation of a consumption plan only slightly, the economically appropriate notion of closeness between consumption plans is the Prohorov distance

$$
d_{\mathcal{M}_{+}}\left(F, F^{\prime}\right) \triangleq \inf \left\{\epsilon>0 \mid F(t-\epsilon)-\epsilon \leq F^{\prime}(t) \leq F(t+\epsilon)+\epsilon \text { for all } t \in[0, T]\right\} .
$$

This metric induces the weak topology on $\mathcal{M}_{+}$. In so far we follow, in spirit if not literally, the fundamental work of Hindy, Huang, and Kreps (1992). 
Under uncertainty, an agent chooses a random element $C(\omega) \in \mathcal{M}_{+}$which has to conform to the available information in the sense that $(C(t))_{0 \leq t \leq T}$ is an adapted process. We assume in addition that expected total consumption remains finite and, thus, the consumption space is

$$
\mathcal{X}_{+} \triangleq\left\{C:\left(\Omega, \mathcal{F}_{T}\right) \rightarrow \mathcal{M}_{+} \mid(C(t))_{0 \leq t \leq T} \text { is adapted with } \mathbb{E} C(T)<+\infty\right\} .
$$

A natural extension of the Prohorov distance to the uncertain framework is given by

$$
d_{\mathcal{X}_{+}}\left(C, C^{\prime}\right) \triangleq \mathbb{E}\left[d_{\mathcal{M}_{+}}\left(C, C^{\prime}\right) \wedge 1\right]+\mathbb{E}\left|C(T)-C^{\prime}(T)\right|
$$

This metric endows $\mathcal{X}_{+}$with the topology of weak convergence in probability plus $L^{1-}$ convergence of total masses.

The consumption space $\mathcal{X}_{+}$has a natural ordering $\preceq$ given by

$$
C \preceq C^{\prime} \Leftrightarrow C^{\prime}-C \in \mathcal{X}_{+} .
$$

Remark 1.1 Note that $\preceq$ does not denote a preference relation. Throughout this paper, agents' preferences will be described by utility functionals. Hence, no confusion should arise.

Every bounded measurable process $\psi: \Omega \times[0, T] \rightarrow \mathbb{R}$ gives rise to a (not necessarily continuous) linear functional $\langle\psi,$.$\rangle on \mathcal{X}_{+}$via

$$
\langle\psi, X\rangle \triangleq \mathbb{E} \int_{0}^{T} \psi(t) d X(t) \quad\left(X \in \mathcal{X}_{+}\right) .
$$

If, in addition, $\psi$ is nonnegative and optional, we call it a price process, and we call $\langle\psi,$.$\rangle a price functional on \mathcal{X}_{+}$.

Remark 1.2 Note that we do not assume continuity of price functionals a priori as is usually done in equilibrium theory. In fact, we will first identify an equilibrium price functional in the much larger space of all linear functionals and establish a posteriori its continuity under appropriate conditions; see Theorem 4.1.

\subsection{Primitives of the Economy}

Each agent $i \in \mathcal{I}$ is endowed with some cumulative income stream $E_{i} \in \mathcal{X}_{+}$. To avoid trivial cases, we assume $E_{i} \neq 0$. Aggregate endowment is $E \triangleq \sum_{i} E_{i}$.

Assumption 1 Agent $i$ 's preferences are described by a utility functional $V_{i}: \mathcal{X}_{+} \rightarrow \mathbb{R}$ with the following properties:

(i) $V_{i}$ is continuous with respect to $d_{\mathcal{X}_{+}}$, strictly concave and strictly increasing with respect to $\preceq$, 
(ii) for every $C \in \mathcal{X}_{+}$there exists a bounded optional process $\nabla V_{i}(C)$ with the subgradient property

$$
V_{i}\left(C^{\prime}\right)-V_{i}(C) \leq\left\langle\nabla V_{i}(C), C^{\prime}-C\right\rangle \quad\left(C^{\prime} \in \mathcal{X}_{+}\right) .
$$

These subgradients are continuous in the sense that, for any two $C, C^{\prime} \in \mathcal{X}_{+}$,

$$
\lim _{\epsilon \downarrow 0}\left\langle\nabla V_{i}\left(\epsilon C^{\prime}+(1-\epsilon) C\right), C^{\prime}-C\right\rangle=\left\langle\nabla V_{i}(C), C^{\prime}-C\right\rangle
$$

Hence, we assume essentially convexity of preferences and a sufficient degree of smoothness. In addition, we require the following technical

Assumption 2 Subgradients are uniformly bounded from above and bounded away from zero in the sense that there are nonnegative, optional processes $b, B \in L_{+}^{1}(\mathbb{P} \otimes d E) \backslash\{0\}$ such that

$$
b \leq \nabla V_{i}(C) \leq B \quad \mathbb{P} \otimes d E-a . e .
$$

for all $C \in \mathcal{X}_{+}$with $C \preceq E$.

\subsection{Examples}

A class of utility functionals which satisfy Assumptions 1 and 2, and which in fact motivated this work are

Hindy-Huang-Kreps Preferences. These preferences are given by a utility functional of the form

$$
V(C) \triangleq \mathbb{E} \int_{0}^{T} u(t, Y(C)(t)) d t \quad\left(C \in \mathcal{X}_{+}\right)
$$

where $u:[0, T] \times \mathbb{R}_{+} \rightarrow \mathbb{R}$ denotes a continuous felicity function which is strictly concave, twice continuously differentiable and increasing in its second argument. The quantity

$$
Y(C)(t) \triangleq \eta e^{-\beta t}+\int_{0}^{t} \beta e^{-\beta(t-s)} d C(s)
$$

describes the investor's level of satisfaction obtained from his consumption up to time $t \in[0, T] ; \eta>0$ is his initial level of satisfaction, and $\beta>0$ measures how fast satisfaction decays.

The subgradient $\nabla V$ at a given consumption plan $C \in \mathcal{X}_{+}$is given by the optional version of

$$
\nabla V(C)(t) \triangleq \mathbb{E}\left[\int_{t}^{T} \partial_{y} u(s, Y(C)(s)) \beta e^{-\beta(s-t)} d s \mid \mathcal{F}_{t}\right] \quad(0 \leq t \leq T)
$$

as has already been shown by Duffie and Skiadas (1994); Bank and Riedel (2000) prove continuity of $\nabla V$ in the sense of the second part of Assumption 1 (ii). The gradient bounds of Assumption 2 can be chosen as

$$
b \triangleq \min _{i} \nabla V_{i}(E) \quad \text { and } \quad B \triangleq \max _{i} \nabla V_{i}(0) .
$$


Remark 1.3 The above Hindy-Huang-Kreps utility is strictly increasing and strictly concave only on the slightly smaller consumption space $\left\{C \in \mathcal{X}_{+} \mid \Delta C(T)=0\right\}$, since consumption made at time $t=T$ obviously does not contribute to agent's utility. However, this minor deviation from Assumption 1 does not impose any problems if we assume $\Delta E(T)=0$.

The generalization of Hindy, Huang, and Zhu (1997) based on two levels of satisfaction is a further example.

Stochastic Differential Utility. Under suitable assumptions, also the stochastic differential utility version of Hindy-Huang-Kreps preferences forms an example for our setup. Indeed, let $f:[0, T] \times \mathbb{R}_{+} \times \mathbb{R} \rightarrow \mathbb{R}$ be a continuous function with the following properties:

(i) for $s \in[0, T], f(s, \cdot, \cdot)$ is strictly increasing, strictly concave, and continuously differentiable,

(ii) for $s \in[0, T]$ and $y \in \mathbb{R}_{+}, f(s, y, \cdot)$ is Lipschitz,

(iii) for $(s, y, w) \in[0, T] \times \mathbb{R}_{+} \times \mathbb{R}$, the derivative with respect to $y$ satisfies

$$
\partial_{y} f(s, y, w) \leq K(1+y)
$$

for some constant $K>0$.

Using similar arguments as in Duffie and Epstein (1992), one obtains for any $C \in \mathcal{X}_{+}$ a unique optional process $U(C)$ such that

$$
U(C)(t)=\mathbb{E}\left[\int_{t}^{T} f(s, Y(C)(s), U(C)(s)) \beta e^{-\beta(s-t)} d s \mid \mathcal{F}_{t}\right] \quad(0 \leq t \leq T)
$$

with $Y(C)$ as above. The utility functional given by $V(C) \triangleq U(C)(0)$ satisfies then Assumption 1; compare Duffie and Skiadas (1994) and Duffie and Epstein (1992). The subgradient takes the form

$$
\nabla V(C)(t)=\mathbb{E}\left[\int_{t}^{T} e^{\delta(s)} \partial_{y} f(s, Y(C)(s), U(C)(s)) \beta e^{-\beta(s-t)} d s \mid \mathcal{F}_{t}\right]
$$

where $\delta(t) \triangleq \int_{0}^{t} \partial_{W} f(s, Y(C)(s), W(C)(s)) d s(0 \leq t \leq T)$.

Remark 1.4 Standard time-additive utility functionals like

$$
V(C)=\mathbb{E} \int_{0}^{T} u(t, c(t)) d t
$$

for absolutely continuous $C(t)=\int_{0}^{t} c(t) d t$ are not continuous with respect to $d_{\mathcal{X}_{+}}$and are thus no examples for our setup; compare Hindy, Huang, and Kreps (1992). 


\subsection{Equilibrium}

An allocation is a vector $\left(C_{i}\right)_{i \in \mathcal{I}} \in \mathcal{X}_{+}^{\mathcal{I}}$. It is feasible if $\sum_{i} C_{i} \preceq E$. The set of feasible allocations will be denoted by $\mathcal{Z}$.

An (Arrow-Debreu) equilibrium consists of a feasible allocation $\left(C_{i}^{*}\right)_{i \in \mathcal{I}} \in \mathcal{Z}$ and a price process $\psi^{*}$ such that, for any $i \in \mathcal{I}$, the consumption plan $C_{i}^{*}$ maximizes agent $i$ 's utility over all $C_{i}$ satisfying the budget-constraint $\left\langle\psi^{*}, C_{i}\right\rangle \leq\left\langle\psi^{*}, E_{i}\right\rangle$.

The main theorem of this paper is

Theorem 1.5 Under Assumptions 1 and 2, an equilibrium exists.

Our strategy of proof is based on the Negishi method as usual with infinite dimensional commodity spaces. In Section 2.1, we first characterize the unique efficient allocation for any given vector of agents' weights. Existence of efficient allocations is ensured by Komlós theorem. The same theorem is also useful in showing that the efficient allocation depends continuously on agents' weights.

In Section 2.2, we characterize the price functionals which support the efficient allocations on the order ideal. The corresponding price processes are given as maxima of agents' utility gradients at the efficient allocation. This particular structure allows us to extend the supporting price functionals from the order ideal to the whole consumption space.

Finally, we define in Section 3 the usual excess utility correspondence. Upper hemicontinuity of this correspondence follows from a classical argument due to Bewley (1969), and Kakutani's fixed point theorem yields existence of an equilibrium.

\section{Efficient Allocations and Supporting Prices}

The first step in our program is to characterize efficient allocations. To this end, we prove a version of the Kuhn-Tucker theorem for the welfare maximization problem. As usual, if agents consume, their utility gradients are equalized in an efficient allocation. The common value for the utility gradients is the Lagrange multiplier for this problem. At the same time, it gives rise to a price functional which supports the efficient allocation in the sense of the Second Welfare Theorem. Of course, their may be other functionals with the same supporting property. However, as we shall see, they all share the same structure.

\subsection{The Social Welfare-Problem}

Let us introduce the set of normalized weights

$$
\Lambda \triangleq\left\{\lambda \in \mathbb{R}_{+}^{\mathcal{I}} \mid \sum_{i} \lambda_{i}=1\right\} .
$$

An allocation $\left(C_{i}\right)_{i \in \mathcal{I}}$ is called $\lambda$-efficient for agents' weights $\lambda \in \Lambda$ if it maximizes the social welfare $\sum_{i} \lambda_{i} V_{i}\left(C_{i}\right)$ subject to the feasibility constraint

$$
\sum_{i} C_{i} \preceq E .
$$


The characterization of efficient allocations is achieved by the following KuhnTucker-like result.

Lemma 2.1 For any $\lambda \in \Lambda$, there exists a unique $\lambda$-efficient allocation $\left(C_{i}^{\lambda}\right)_{i \in \mathcal{I}} \in \mathcal{Z}$. It is characterized by the joint validity of the following properties (i)-(iii) for some nonnegative, optional random variable $\psi$ :

(i) $\sum_{i} C_{i}^{\lambda}=E$,

(ii) $\lambda_{i} \nabla V_{i}\left(C_{i}^{\lambda}\right) \leq \psi$,

(iii) $\left\langle\psi-\lambda_{i} \nabla V_{i}\left(C_{i}^{\lambda}\right), C_{i}^{\lambda}\right\rangle=0$ for every $i \in \mathcal{I}$.

The random variable $\psi$ plays the role of a Lagrange multiplier for the problem of maximizing social welfare. By the flat-off condition (iii), it is uniquely determined $\mathbb{P} \otimes d E$-almost everywhere as

$$
\psi=\max _{i}\left\{\lambda_{i} \nabla V_{i}\left(C_{i}^{\lambda}\right)\right\}
$$

Proof : Uniqueness of the $\lambda$-efficient allocation follows as usual from the strict concavity of the utility functionals $V_{i}(i \in \mathcal{I})$ by considering a convex combination of two $\lambda$-efficient allocations.

To prove existence, we choose a welfare-maximizing sequence of feasible allocations $\left(\left(C_{i}^{n}\right)_{i \in \mathcal{I}}, n=1,2, \ldots\right)$ such that

$$
\sum_{i} \lambda_{i} V_{i}\left(C_{i}^{n}\right) \rightarrow \sup _{\left(C_{i}\right) \in \mathcal{Z}} \sum_{i} \lambda_{i} V_{i}\left(C_{i}\right)
$$

By feasibility, each sequence $\left(C_{i}^{n}(T), n=1,2, \ldots\right)(i \in \mathcal{I})$ is bounded in $L^{1}(\mathbb{P})$. Hence, we may use Kabanov's version of Komlós' theorem (Kabanov (1999), Lemma 3.5; Komlós (1967)) to obtain existence of a subsequence, again denoted by $n$, such that each sequence $\left(C_{i}^{n}, n=1,2, \ldots\right)(i \in \mathcal{I})$ is almost surely weakly Cesaro convergent to some $C_{i}^{*}(i \in \mathcal{I})$, i.e., we have almost surely

$$
\tilde{C}_{i}^{n}(t) \triangleq \frac{1}{n} \sum_{k=1}^{n} C_{i}^{k}(t) \rightarrow C_{i}^{*}(t)
$$

for $t=T$ and also for every point of continuity $t$ of $C_{i}^{*}$. The above convergence shows that $\left(C_{i}^{*}\right)_{i \in \mathcal{I}}$ also is a feasible allocation. Moreover, it implies $d_{\mathcal{X}_{+}}\left(\tilde{C}_{i}^{n}, C_{i}^{*}\right) \rightarrow 0$, as, in addition to the above weak convergence, $\left(\tilde{C}_{i}^{n}(T), n=1,2, \ldots\right)$ is dominated since

$$
0 \leq \tilde{C}_{i}^{n}(T) \leq E(T)
$$

by feasibility. Finally, also $\left(\left(\tilde{C}_{i}^{n}\right)_{i \in \mathcal{I}}, n=1,2, \ldots\right)$ is a maximizing sequence of feasible allocations due to concavity of social welfare. As $\tilde{C}_{i}^{n} \rightarrow C_{i}^{*}$ in $\left(\mathcal{X}_{+}, d_{\mathcal{X}_{+}}\right)$for every $i \in \mathcal{I}$, this implies $\lambda$-efficiency of $\left(C_{i}^{*}\right)_{i \in \mathcal{I}}$ by continuity of preferences (Assumption 1 (i)).

In order to prove the asserted characterization of efficient allocations, we proceed in three steps. 
1. We start with sufficiency of (i)-(iii). Let $\left(C_{i}^{\lambda}\right)_{i \in \mathcal{I}}$ denote the $\lambda$-efficient allocation and let $\left(C_{i}\right)_{i \in \mathcal{I}} \in \mathcal{Z}$ be another feasible allocation. Due to the subgradient estimate of Assumption 1 (ii), we have

$$
\sum_{i} \lambda_{i}\left\{V_{i}\left(C_{i}^{\lambda}\right)-V_{i}\left(C_{i}\right)\right\} \geq \sum_{i} \lambda_{i}\left\langle\nabla V_{i}\left(C_{i}^{\lambda}\right), C_{i}^{\lambda}-C_{i}\right\rangle
$$

which by (ii) and (iii) is

$$
\geq\left\langle\psi, \sum_{i} C_{i}^{\lambda}-\sum_{i} C_{i}\right\rangle
$$

As $\psi$ is nonnegative, this yields

$$
\sum_{i} \lambda_{i}\left\{V_{i}\left(C_{i}^{\lambda}\right)-V_{i}\left(C_{i}\right)\right\} \geq\left\langle\psi, E-\sum_{i} C_{i}\right\rangle \geq 0
$$

by condition (i) and feasibility of $\left(C_{i}\right)_{i \in \mathcal{I}}$. Hence an allocation $\left(C_{i}\right)_{i \in \mathcal{I}}$ with (i)-(iii) indeed attains maximal social welfare among all feasible allocations, given agents' weights $\lambda$.

2. Necessity of condition (i) follows immediately from strict monotonicity of preferences. To prove that conditions (ii) and (iii) hold true for some Lagrange parameter $\psi$, consider another feasible allocation $\left(C_{i}\right)_{i \in \mathcal{I}} \in \mathcal{Z}$. For $\epsilon \in[0,1]$, let $C_{i}^{\epsilon} \triangleq \epsilon C_{i}+(1-\epsilon) C_{i}^{\lambda}(i \in \mathcal{I})$. Since every allocation $\left(C_{i}^{\epsilon}\right)_{i \in \mathcal{I}}$ is feasible, $\lambda$-efficiency of $\left(C_{i}^{\lambda}\right)_{i \in \mathcal{I}}$ yields, for $\epsilon \in(0,1]$,

$$
\begin{aligned}
0 & \geq \frac{1}{\epsilon} \sum_{i} \lambda_{i}\left\{V_{i}\left(C_{i}^{\epsilon}\right)-V_{i}\left(C_{i}^{\lambda}\right)\right\} \\
& \geq \frac{1}{\epsilon} \sum_{i} \lambda_{i}\left\langle\nabla V_{i}\left(C_{i}^{\epsilon}\right), C_{i}^{\epsilon}-C_{i}^{\lambda}\right\rangle=\sum_{i} \lambda_{i}\left\langle\nabla V_{i}\left(C_{i}^{\epsilon}\right), C_{i}-C_{i}^{\lambda}\right\rangle .
\end{aligned}
$$

Using continuity of subgradients (Assumption 1 (ii)), we may let $\epsilon \downarrow 0$ in the above estimate to deduce

$$
\sum_{i}\left\langle\phi_{i}, C_{i}\right\rangle \leq \sum_{i}\left\langle\phi_{i}, C_{i}^{\lambda}\right\rangle
$$

where $\phi_{i} \triangleq \lambda_{i} \nabla V_{i}\left(C_{i}^{\lambda}\right)(i \in \mathcal{I})$.

We see that $\left(C_{i}^{\lambda}\right)_{i \in \mathcal{I}}$ also solves the linear problem to maximize $\sum_{i}\left\langle\phi_{i}, C_{i}\right\rangle$ over all feasible allocations. In Step 3 below, we show that every solution $\left(C_{i}^{*}\right)_{i \in \mathcal{I}}$ of this problem satisfies $\left\langle\psi-\phi_{i}, C_{i}^{*}\right\rangle=0$ for every $i \in \mathcal{I}$ where $\psi \triangleq \max _{i} \phi_{i}$. For $C^{*}=C^{\lambda}$, we find that, with this choice of $\psi$, conditions (ii) and (iii) in fact hold true, too. 
3. Let $\left(C_{i}^{*}\right)_{i \in \mathcal{I}} \in \mathcal{Z}$ be a feasible allocation such that

$$
\sum_{i}\left\langle\phi_{i}, C_{i}\right\rangle \leq \sum_{i}\left\langle\phi_{i}, C_{i}^{*}\right\rangle
$$

for every other feasible allocation $\left(C_{i}\right)_{i \in \mathcal{I}} \in \mathcal{Z}$.

Consider the allocation defined by the optional random measures

$$
d C_{i}(t) \triangleq n(t)^{-1} 1_{\left\{\phi_{i}(t)=\psi(t)\right\}} d E(t) \quad(i \in \mathcal{I})
$$

with $\psi(t)=\max _{i} \phi_{i}(t)$ and where $n(t) \triangleq \sum_{i} 1_{\left\{\phi_{i}(t)=\psi(t)\right\}}$ denotes the number of indices $i$ realizing the $\max$ in $\max _{i} \phi_{i}(t)(0 \leq t \leq T)$. Clearly, $\left(C_{i}\right)_{i \in \mathcal{I}}$ is feasible and satisfies

$$
\sum_{i}\left\langle\phi_{i}, C_{i}\right\rangle=\langle\psi, E\rangle
$$

Therefore, $\sum_{i}\left\langle\phi_{i}, C_{i}^{*}\right\rangle$ cannot be less than $\langle\psi, E\rangle$. On the other hand, since $\psi \geq$ $\phi_{i} \geq 0$ for each $i \in \mathcal{I}$ and as $\left(C_{i}^{*}\right)_{i \in \mathcal{I}}$ is feasible, $\sum_{i}\left\langle\phi_{i}, C_{i}^{*}\right\rangle$ cannot be greater than $\langle\psi, E\rangle$. Thus, we must in fact have

$$
\sum_{i}\left\langle\phi_{i}, C_{i}^{*}\right\rangle=\langle\psi, E\rangle
$$

which can hold true only if $\left\langle\psi-\phi_{i}, C_{i}^{*}\right\rangle=0$ for every $i \in \mathcal{I}$.

The dependence of efficient allocations and induced expected utilities on agents' weights will be described in the following Lemma 2.2 and its Corollary 2.3. The latter result will be a corner stone for our proof of Theorem 1.5.

Lemma 2.2 The mapping $\lambda \mapsto C^{\lambda}$ is weakly continuous in the sense that

$$
\lim _{\lambda^{n} \rightarrow \lambda^{0}}\left\langle\psi, C_{i}^{\lambda^{n}}\right\rangle=\left\langle\psi, C_{i}^{\lambda^{0}}\right\rangle \quad(i \in \mathcal{I})
$$

for every $\psi \in L^{1}(\mathbb{P} \otimes d E)$.

Proof : Let $\lambda^{n}(n=1,2, \ldots)$ tend to $\lambda^{0}$ in $\Lambda$. Put $C^{n} \triangleq C^{\lambda^{n}}$ and consider the densities

$$
D_{i}^{n} \triangleq \frac{d C_{i}^{n}}{d E} \quad(n=0,1, \ldots, i \in \mathcal{I}) .
$$

Due to feasibility of efficient allocations, these densities have optional versions taking values in $[0,1]$. Now, writing

$$
\left\langle\psi, C_{i}^{n}\right\rangle=\mathbb{E} \int_{0}^{T} \psi(t) D_{i}^{n}(t) d E(t) \quad(n=0,1, \ldots i \in \mathcal{I})
$$

for $\psi \in L^{1}(\mathbb{P} \otimes d E)$, we see that our assertion is equivalent to the assertion that, for every $i \in \mathcal{I}$ the densities $D_{i}^{n}(n=1,2, \ldots)$ converge to $D_{i}^{0}$ in the weak ${ }^{*}$-topology 
$\sigma\left(L^{\infty}(\mathbb{P} \otimes d E), L^{1}(\mathbb{P} \otimes d E)\right)$. As the unit ball in $L^{\infty}(\mathbb{P} \otimes d E)$ is sequentially compact with respect to this topology, this convergence will be proved once we know that, for each $i \in \mathcal{I}$, all weak ${ }^{*}$-convergent subsequences of $\left(D_{i}^{n}, n=1,2, \ldots\right)$ have the same limit $D_{i}^{0}$.

To prove this, we slightly abuse notation and suppose that $\left(\left(D_{i}^{n}\right)_{i \in \mathcal{I}}, n=1,2, \ldots\right)$ is a subsequence such that each component $\left(D_{i}^{n}, n=1,2, \ldots\right)$ is weak ${ }^{*}$-convergent to some $D_{i}^{\infty} \in L^{\infty}(\mathbb{P} \otimes d E)(i \in \mathcal{I})$. We then have to show that $D_{i}^{\infty}=D_{i}^{0}$ for every $i \in \mathcal{I}$.

For this, we note first that, by feasibility, we have $\mathbb{E} C_{i}^{n}(T) \leq \mathbb{E} E(T)$ for any $n=$ $1,2, \ldots$ and every $i \in \mathcal{I}$. Hence, by Kabanov's version of Komlós' theorem, there is a subsequence, again denoted by $\left(\left(C_{i}^{n}\right)_{i \in \mathcal{I}}, n=1,2, \ldots\right)$, such that, for any $i \in \mathcal{I}$,

$$
\tilde{C}_{i}^{n} \triangleq \frac{1}{n} \sum_{k=1}^{n} C_{i}^{k}
$$

almost surely converges weakly to some $C_{i}^{*} \in \mathcal{X}_{+}$in the sense that

$$
d_{\mathcal{M}_{+}}\left(\tilde{C}_{i}^{n}, C_{i}^{*}\right) \rightarrow 0 \quad(n \uparrow+\infty)
$$

By dominated convergence, we also have $\tilde{C}_{i}^{n}(T) \rightarrow C_{i}^{*}(T)$ in $L^{1}(\mathbb{P})$ and, therefore, even $d_{\mathcal{X}_{+}}\left(\tilde{C}_{i}^{n}, C_{i}^{*}\right) \rightarrow 0$ for every $i \in \mathcal{I}$. Clearly, the densities

$$
\tilde{D}_{i}^{n} \triangleq \frac{d \tilde{C}_{i}^{n}}{d E}=\frac{1}{n} \sum_{k=1}^{n} D_{i}^{k} \quad(n=1,2, \ldots)
$$

inherit weak ${ }^{*}$-convergence to $D_{i}^{\infty}$ from $\left(D_{i}^{n}, n=1,2, \ldots\right)$. Therefore, each $C_{i}^{*}$ is almost surely absolutely continuous with respect to $E$ with density $D_{i}^{\infty}$. Hence, in order to conclude our claim, we only need to prove that $\left(C_{i}^{*}\right)_{i \in \mathcal{I}}$ is the unique (!) efficient allocation for agents' weights $\lambda^{0}$.

To this end, consider any other feasible allocation $C=\left(C_{i}\right)_{i \in \mathcal{I}} \in \mathcal{Z}$. By continuity of preferences, we have

$$
\sum_{i} \lambda_{i}^{0} V_{i}\left(C_{i}^{*}\right)=\lim _{n} \sum_{i} \lambda_{i}^{0} V_{i}\left(\tilde{C}_{i}^{n}\right)
$$

which, due to the concavity of every $V_{i}().(i \in \mathcal{I})$, is

$$
\begin{aligned}
& \geq \underset{n}{\limsup } \frac{1}{n} \sum_{k=1}^{n} \sum_{i} \lambda_{i}^{0} V_{i}\left(C_{i}^{k}\right) \\
& =\underset{n}{\limsup } \frac{1}{n} \sum_{k=1}^{n} \sum_{i}\left\{\lambda_{i}^{k} V_{i}\left(C_{i}^{k}\right)+R_{i}^{k}\right\}
\end{aligned}
$$

for $R_{i}^{k} \triangleq\left(\lambda_{i}^{0}-\lambda_{i}^{k}\right) V_{i}\left(C_{i}^{k}\right)(k=1,2, \ldots, i \in \mathcal{I})$. This term tends to zero for $k \uparrow \infty$ as $V_{i}\left(C_{i}^{k}\right) \in\left[V_{i}(0), V_{i}(E)\right]$ is uniformly bounded and $\lambda^{k} \rightarrow \lambda^{0}(k \uparrow \infty)$. Hence, we obtain

$$
\sum_{i} \lambda_{i}^{0} V_{i}\left(C_{i}^{*}\right) \geq \limsup _{n} \frac{1}{n} \sum_{k=1}^{n} \sum_{i} \lambda_{i}^{k} V_{i}\left(C_{i}^{k}\right)
$$


By $\lambda^{k}$-efficiency of allocation $C^{k}(k=1,2, \ldots)$, this is in turn

$$
\begin{aligned}
& \geq \underset{n}{\limsup } \frac{1}{n} \sum_{k=1}^{n} \sum_{i} \lambda_{i}^{k} V_{i}\left(C_{i}\right) \\
& =\underset{n}{\limsup } \sum_{i}\left(\frac{1}{n} \sum_{k=1}^{n} \lambda_{i}^{k}\right) V_{i}\left(C_{i}\right) \\
& =\sum_{i} \lambda_{i}^{0} V_{i}\left(C_{i}\right)
\end{aligned}
$$

where the last equality is due to the convergence $\lambda^{n} \rightarrow \lambda^{0}$. Hence, we have shown

$$
\sum_{i} \lambda_{i}^{0} V_{i}\left(C_{i}^{*}\right) \geq \sum_{i} \lambda_{i}^{0} V_{i}\left(C_{i}\right)
$$

for every feasible allocation $\left(C_{i}\right)_{i \in \mathcal{I}}$. Since, in addition, $\left(C_{i}^{*}\right)_{i \in \mathcal{I}}$ is feasible, it must coincide with the unique $\lambda^{0}$-efficient allocation $\left(C_{i}^{0}\right)_{i \in \mathcal{I}}$ and we are done.

Let us note the following crucial

Corollary 2.3 Every mapping $\lambda \mapsto V_{i}\left(C_{i}^{\lambda}\right)(i \in \mathcal{I})$ is upper-semicontinuous, i.e.,

$$
\limsup _{\lambda^{n} \rightarrow \lambda^{0}} V_{i}\left(C_{i}^{\lambda^{n}}\right) \leq V_{i}\left(C_{i}^{\lambda^{0}}\right) .
$$

Proof : By concavity we have

$$
V_{i}\left(C_{i}^{\lambda^{n}}\right)-V_{i}\left(C_{i}^{\lambda^{0}}\right) \leq\left\langle\nabla V_{i}\left(C_{i}^{\lambda^{0}}\right), C_{i}^{\lambda^{n}}-C_{i}^{\lambda^{0}}\right\rangle .
$$

By Lemma 2.2, the last term tends to zero if $\lambda^{n} \rightarrow \lambda^{0}$ in $\Lambda$ and we are done.

\subsection{Supporting Prices}

For some of the following more technical arguments, it will be convenient to work with the auxiliary consumption space given by the order ideal

$$
\tilde{\mathcal{X}}_{+}=\tilde{\mathcal{X}}_{+}(E) \triangleq\left\{X \in \mathcal{X}_{+} \mid \frac{d X}{d E} \text { exists } \mathbb{P} \text {-a.s. and is } \mathbb{P} \otimes d E \text {-essentially bounded }\right\} .
$$

For a general discussion of the order ideal in general equilibrium theory, we refer the reader to Mas-Colell and Zame (1991) .

Remark 2.4 The space $\tilde{\mathcal{X}}_{+}$can be identified with $L_{+}^{\infty}(\Omega \times[0, T], \mathcal{O}, \mathbb{P} \otimes d E)$, the set of all nonnegative, optional processes which are $\mathbb{P} \otimes d E$-essentially bounded. Clearly, any feasible allocation is contained in $\tilde{\mathcal{X}}_{+}^{\mathcal{I}}$. 
By Lemma 2.1, we may associate to every efficient allocation $\left(C_{i}^{\lambda}\right)_{i \in \mathcal{I}}(\lambda \in \Lambda)$ the Lagrange multiplier

$$
\psi_{\lambda} \triangleq \max _{i}\left\{\lambda_{i} \nabla V_{i}\left(C_{i}^{\lambda}\right)\right\}
$$

We will have a lot more to say about the structure of these multipliers in Section 4 . For the moment, let us content ourselves by noting that each $\psi_{\lambda}$ can be viewed as a nonnegative, optional random variable in $L^{1}(\mathbb{P} \otimes d E)$. Hence, each of these Lagrange multipliers gives rise to a price density on $\tilde{\mathcal{X}}_{+}$. Considering $\psi_{\lambda}$ as a price density is also sustained by the fact that it supports its associated efficient allocation $\left(C_{i}^{\lambda}\right)_{i \in \mathcal{I}}$. This will be proved in Lemma 2.6 below.

Beforehand, let us recall that a price density $\psi^{*} \in L^{1}(\mathbb{P} \otimes d E)$ supports an allocation $\left(C_{i}^{*}\right)_{i \in \mathcal{I}}$ with $\sum_{i} C_{i}^{*}=E$, if it is non-zero and if any preferred allocation $\left(C_{i}\right)_{i \in \mathcal{I}} \in \tilde{\mathcal{X}}_{+}^{\mathcal{I}}$ has a higher 'price' under $\psi^{*}$ than $\left(C_{i}^{*}\right)_{i \in \mathcal{I}}$. More precisely, we say $\psi^{*} \operatorname{supports}\left(C_{i}^{*}\right)_{i \in \mathcal{I}}$ with $\sum_{i} C_{i}^{*}=E$ iff $\left\langle\psi^{*}, E\right\rangle \neq 0$ and

$$
V_{i}\left(C_{i}\right) \geq V_{i}\left(C_{i}^{*}\right), C_{i} \in \tilde{\mathcal{X}}_{+} \text {for all } i \in \mathcal{I} \text { implies }\left\langle\psi^{*}, \sum_{i} C_{i}\right\rangle \geq\left\langle\psi^{*}, E\right\rangle
$$

We note the following

Proposition 2.5 If an allocation $\left(C_{i}^{*}\right)_{i \in \mathcal{I}}$ with $\sum_{i} C_{i}^{*}=E$ is supported by $\psi^{*} \in L^{1}(\mathbb{P} \otimes$ $E)$, then $\psi^{*}>0 \mathbb{P} \otimes d E-$ a.e.

Proof : Suppose to the contrary that $\mathbb{P} \otimes d E\left[\psi^{*} \leq 0\right]>0$. Then, by strict monotonicity and continuity of preferences, we may choose $\epsilon>0$ small enough such that the allocation defined by

$$
d C_{i} \triangleq(1-\epsilon) d C_{i}^{*}+1_{\left\{\psi^{*} \leq 0\right\}} d E \quad(i \in \mathcal{I})
$$

is preferred to $\left(C_{i}^{*}\right)_{i \in \mathcal{I}}$. Thus, the supporting property of $\psi^{*}$ implies

$$
\left\langle\psi^{*}, E\right\rangle \leq\left\langle\psi^{*}, \sum_{i} C_{i}\right\rangle=(1-\epsilon)\left\langle\psi^{*}, E\right\rangle+|\mathcal{I}| \mathbb{E} \int_{0}^{T} \psi^{*} 1_{\left\{\psi^{*} \leq 0\right\}} d E .
$$

Hence, we obtain

$$
|\mathcal{I}| \mathbb{E} \int_{0}^{T} \psi^{*} 1_{\left\{\psi^{*} \leq 0\right\}} d E \geq \epsilon\left\langle\psi^{*}, E\right\rangle>0
$$

a contradiction to the obvious relation $\mathbb{E} \int_{0}^{T} \psi^{*} 1_{\left\{\psi^{*} \leq 0\right\}} d E \leq 0$.

Now, we may prove

Lemma 2.6 The Lagrange multiplier $\psi_{\lambda}$ supports its associated $\lambda$-efficient allocation $\left(C_{i}^{\lambda}\right)_{i \in \mathcal{I}}$. Moreover, any other optional price density $\psi \in L^{1}(\mathbb{P} \otimes d E)$ with this property is of the form

$$
\psi=\max _{i}\left\{k_{i} \nabla V_{i}\left(C_{i}^{\lambda}\right)\right\} \quad \mathbb{P} \otimes d E-a . s .
$$


for some constants $k_{i} \geq 0(i \in \mathcal{I})$, and the $\lambda$-efficient allocation satisfies the flat-off conditions

$$
\left\langle\psi-k_{i} \nabla V_{i}\left(C_{i}^{\lambda}\right), C_{i}^{\lambda}\right\rangle=0 \quad(i \in \mathcal{I})
$$

ProOF : In order to show that $\psi_{\lambda}$ supports $\left(C_{i}^{\lambda}\right)_{i \in \mathcal{I}}$, take an allocation $\left(C_{i}\right)_{i \in \mathcal{I}} \in \tilde{\mathcal{X}}_{+}^{\mathcal{I}}$ with $V_{i}\left(C_{i}\right) \geq V_{i}\left(C_{i}^{\lambda}\right)$. Concavity of $V_{i}$ yields

$$
0 \leq \sum_{i} \lambda_{i}\left\{V_{i}\left(C_{i}\right)-V_{i}\left(C_{i}^{\lambda}\right)\right\} \leq \sum_{i} \lambda_{i}\left\langle\nabla V_{i}\left(C_{i}^{\lambda}\right), C_{i}-C_{i}^{\lambda}\right\rangle .
$$

By properties (ii) and (iii) of the efficient allocation $\left(C_{i}^{\lambda}\right)_{i \in \mathcal{I}}$ (Lemma 2.1), the latter quantity is

$$
\leq \sum_{i}\left\langle\psi_{\lambda}, C_{i}-C_{i}^{\lambda}\right\rangle=\left\langle\psi_{\lambda}, \sum_{i} C_{i}-E\right\rangle
$$

Hence, $\left\langle\psi_{\lambda}, \sum_{i} C_{i}\right\rangle \geq\left\langle\psi_{\lambda}, E\right\rangle$ which is the claimed supporting property.

For the second part of the lemma, suppose that $\psi \in L^{1}(\mathbb{P} \otimes d E)$ is optional and supports the allocation $\left(C_{i}^{\lambda}\right)_{i \in \mathcal{I}}$. We only need to show that there are nonnegative constants $k_{i}(i \in \mathcal{I})$ such that

$$
k_{i} \nabla V_{i}\left(C_{i}^{\lambda}\right) \leq \psi \mathbb{P} \otimes d E-\text { a.e. and }\left\langle\psi-k_{i} \nabla V_{i}\left(C_{i}^{\lambda}\right), C_{i}^{\lambda}\right\rangle=0
$$

for every $i \in \mathcal{I}$. To this end, put

$$
k_{i} \triangleq \mathbb{P} \otimes d E-\operatorname{ess} \inf \frac{\psi}{\nabla V_{i}\left(C_{i}^{\lambda}\right)} \quad(i \in \mathcal{I}) .
$$

Obviously, $k_{i} \geq 0$ for every $i \in \mathcal{I}$ and, of course, the first condition in (1) is satisfied. To verify the second condition for $i \in \mathcal{I}$, let us distinguish the cases $\left\langle\psi, C_{i}^{\lambda}\right\rangle=0$ and $\left\langle\psi, C_{i}^{\lambda}\right\rangle>0$.

In the first case, we may conclude from Proposition 2.5 that $C_{i}^{\lambda}=0$. Thus, the second condition in (1) is satisfied trivially. For the case $\left\langle\psi, C_{i}^{\lambda}\right\rangle>0$, we prove below that $C_{i}^{\lambda}$ maximizes utility over all consumption plans $C_{i} \in \tilde{\mathcal{X}}_{+}$with $\left\langle\psi, C_{i}\right\rangle \leq\left\langle\psi, C_{i}^{\lambda}\right\rangle$. By arguments similar to the proof of Theorem 3.3 in Bank and Riedel (1999), one then deduces the validity of the second condition in (1) also in this case.

To obtain the claimed optimality of $C_{i}^{\lambda}$, it suffices to show that any $C_{i} \in \tilde{\mathcal{X}}_{+}$with $V_{i}\left(C_{i}\right)>V_{i}\left(C_{i}^{\lambda}\right)$ must satisfy $\left\langle\psi, C_{i}\right\rangle>\left\langle\psi, C_{i}^{\lambda}\right\rangle$. Note first that, for such a $C_{i}$, we also have $V_{i}\left((1-\epsilon) C_{i}\right)>V_{i}\left(C_{i}^{\lambda}\right)$ for any sufficiently small $\epsilon>0$ by continuity $V_{i}$. Consider the allocation $\left(\tilde{C}_{j}\right)_{j \in \mathcal{I}}$ defined by

$$
\tilde{C}_{j} \triangleq \begin{cases}(1-\epsilon) C_{i} & \text { for } j=i \\ C_{j}^{\lambda} & \text { otherwise }\end{cases}
$$

This allocation is preferred to $\left(C_{i}^{\lambda}\right)_{i \in \mathcal{I}}$ and, thus, the supporting property of $\psi$ yields

$$
\langle\psi, E\rangle \leq\left\langle\psi, \sum_{j} \tilde{C}_{j}\right\rangle=\langle\psi, E\rangle+\left\langle\psi,(1-\epsilon) C_{i}-C_{i}^{\lambda}\right\rangle
$$


This implies

$$
0<\left\langle\psi, C_{i}^{\lambda}\right\rangle \leq\left\langle\psi,(1-\epsilon) C_{i}\right\rangle<\left\langle\psi, C_{i}\right\rangle
$$

as we wanted to show.

\section{Existence of Equilibria}

After these technical preliminaries, we are now in a position to prove existence of equilibria for intertemporal consumption.

Proof of Theorem 1.5 We start by defining the correspondence $\mathcal{G}$ to which Kakutani's fixed point theorem will be applied. To this end, let, for any $\lambda \in \Lambda, \mathcal{S}(\lambda)$ denote the set of all optional price densities $\psi \in L^{1}(\mathbb{P} \otimes d E)$ which support the allocation $C^{\lambda}$ and which, in addition, satisfy

$$
\frac{b}{|\mathcal{I}|\langle B, E\rangle} \leq \psi \leq \frac{B}{\langle B, E\rangle} \quad \mathbb{P} \otimes d E \text {-a.e. } \quad \text { and } \quad\left\langle\psi, C_{i}^{\lambda}\right\rangle \leq \lambda_{i} \quad \text { for every } i \in \mathcal{I}
$$

Here, $b$ and $B$ are the optional gradient bounds introduced in Assumption 2.

We now define the correspondence

$$
\mathcal{G}(\lambda) \triangleq\left\{\left(\lambda_{i}+\left\langle\psi, E_{i}-C_{i}^{\lambda}\right\rangle\right)_{i \in \mathcal{I}} \mid \psi \in \mathcal{S}(\lambda)\right\} \quad(\lambda \in \Lambda)
$$

In Proposition 3.1 below, we show that indeed $\mathcal{G}$ satisfies the conditions required for Kakutani's theorem. Hence, $\mathcal{G}$ has a fixed point $\lambda^{*} \in \Lambda$. Let $C^{*} \triangleq C^{\lambda^{*}}$ and note that, by definition of $\mathcal{G}$, there is $\psi^{*} \in \mathcal{S}\left(\lambda^{*}\right)$ such that $\left\langle\psi^{*}, E_{i}\right\rangle=\left\langle\psi^{*}, C_{i}^{*}\right\rangle$ for every $i \in \mathcal{I}$. As $\psi^{*}$ supports the efficient allocation $C^{*}$, this gives us existence of a quasi-equilibrium in the auxiliary economy where consumption spaces are given by the order ideal $\tilde{\mathcal{X}}_{+} \subset \mathcal{X}_{+}$.

Now, recall from Lemma 2.6 that, as the density $\psi^{*}$ induces a price functional supporting the allocation $\left(C_{i}^{*}\right)_{i \in \mathcal{I}}$, it must take the form

$$
\psi^{*}=\max _{i}\left\{k_{i}^{*} \nabla V_{i}\left(C_{i}^{*}\right)\right\}
$$

for some constants $k_{i}^{*} \geq 0$. Note that, via the right side of $(4), \psi^{*}$ allows a canonical extension to a strictly positive, bounded and optional process on the whole time interval $[0, T]$. This process induces, thus, a price functional $\left\langle\psi^{*},.\right\rangle$ on the 'large' consumption space $\mathcal{X}_{+}$.

Let us next show that, in conjunction with $\left(C_{i}^{*}\right)_{i \in \mathcal{I}}$, this functional $\left\langle\psi^{*},.\right\rangle$ defines a true Arrow-Debreu-equilibrium for the 'large' economy where consumption spaces are given by $\mathcal{X}_{+}$. To this end, fix $i \in \mathcal{I}$ and consider a plan $C_{i} \in \mathcal{X}_{+}$which is strictly preferred to $C_{i}^{*}$, i.e., assume $V_{i}\left(C_{i}\right)>V_{i}\left(C_{i}^{*}\right)$. We have to show that $\left.\left\langle\psi^{*}, C_{i}\right\rangle\right\rangle\left\langle\psi^{*}, C_{i}^{*}\right\rangle=$ $\left\langle\psi^{*}, E_{i}\right\rangle$. In fact, we have

$$
0>V_{i}\left(C_{i}^{*}\right)-V_{i}\left(C_{i}\right) \geq\left\langle\nabla V_{i}\left(C_{i}^{*}\right), C_{i}^{*}-C_{i}\right\rangle \geq\left\langle\psi^{*}, C_{i}^{*}-C_{i}\right\rangle / k_{i}^{*},
$$


where the last estimate is due to $k_{i}^{*} \nabla V_{i}\left(C_{i}^{*}\right) \leq \psi^{*}$ and to the flat-off condition in Lemma 2.6. Hence, any plan which is strictly preferred to $C_{i}^{*}$ violates the investor's budget constraint.

It remains to establish

Proposition 3.1 The correspondence $\mathcal{G}$ defined by (3) satisfies the conditions of Kakutani's fixed point theorem:

(i) For every $\lambda \in \Lambda, \mathcal{G}(\lambda)$ is a non-empty convex subset of $\Lambda$.

(ii) $\mathcal{G}$ is upper hemi-continuous, i.e., the graph

$$
\{(\lambda, g) \mid \lambda \in \Lambda, g \in \mathcal{G}(\lambda)\}
$$

is closed in $\Lambda \times \Lambda$.

ProOF : We adopt the notation from the preceding proof.

1. Let us focus on assertion (i) and note first that $\mathcal{G}(\lambda)$ is nonempty for every $\lambda \in \Lambda$. Indeed, we know from Lemma 2.6 that $\psi_{\lambda}$ supports the allocation $C^{\lambda}$. Clearly, this property is inherited by every positive multiple of $\psi_{\lambda}$. Moreover, we have

$$
\frac{b}{|\mathcal{I}|} \leq \psi_{\lambda} \leq B
$$

such that $\psi \triangleq \psi_{\lambda} /\langle B, E\rangle$ obviously satisfies the first constraint in (2). It also satisfies the second constraint, since by Lemma 2.1 (iii)

$$
\left\langle\psi_{\lambda}, C_{i}^{\lambda}\right\rangle=\lambda_{i}\left\langle\nabla V_{i}\left(C_{i}^{\lambda}\right), C_{i}^{\lambda}\right\rangle \leq \lambda_{i}\langle B, E\rangle
$$

This shows that $\mathcal{S}(\lambda)$ and, hence, also $\mathcal{G}(\lambda)$ is nonempty.

Convexity of $\mathcal{G}(\lambda)$ follows from convexity of $\mathcal{S}(\lambda)$. Moreover, any $g \in \mathcal{G}(\lambda)$ satisfies $g_{i} \geq 0$ for every $i \in \mathcal{I}$ because of the second constraint in (2). In addition, $\sum_{i} g_{i}=1$ by Lemma 2.1 (i). Hence, we have in fact $\mathcal{G}(\lambda) \subset \Lambda$ which completes the proof of assertion (i).

2. To prove assertion (ii), let $\lambda^{n} \in \Lambda$ and $g^{n} \in \mathcal{G}\left(\lambda^{n}\right)(n=1,2, \ldots)$ converge to $\lambda^{0}$ and $g^{0}$, respectively. We have to show that $g^{0} \in \mathcal{G}\left(\lambda^{0}\right)$. Put $C^{n} \triangleq C^{\lambda^{n}}$ and let $\psi_{n} \in \mathcal{S}\left(\lambda^{n}\right)$ be such that

$$
g_{i}^{n}=\lambda_{i}^{n}+\left\langle\psi_{n}, E_{i}-C_{i}^{n}\right\rangle \quad(i \in \mathcal{I}) .
$$

Due to condition (2), the sequence $\left(\psi_{n}, n=1,2, \ldots\right)$ is dominated by the $\mathbb{P} \otimes d E$ integrable process $B /\langle B, E\rangle$. In particular, it is uniformly integrable and, by the Dunford-Pettis-theorem, there is a subsequence, again denoted by $\left(\psi_{n}\right)$, which converges weakly to some $\psi$ in $L^{1}(\mathbb{P} \otimes d E)$. 
We shall show that $\psi$ belongs to $\mathcal{S}\left(\lambda^{0}\right)$ and satisfies

$$
g_{i}^{0}=\lambda_{i}^{0}+\left\langle\psi, E_{i}-C_{i}^{0}\right\rangle \quad \text { for every } i \in \mathcal{I} .
$$

Of course, this will yield assertion (ii). Our argument is based on the following result

$$
\lim _{n}\left\langle\psi_{n}, C_{i}^{n}\right\rangle=\left\langle\psi, C_{i}^{0}\right\rangle \quad \text { for every } i \in \mathcal{I}
$$

which goes back to Bewley (1969) and which will be established in Step 3 of this proof.

As a first application of Bewley's claim, we note that (6) holds true. Indeed, granted (7), this follows immediately by letting $n \uparrow \infty$ in (5).

Similarly, we show that $\psi$ satisfies the second condition in (2) for $\lambda=\lambda^{0}$. Indeed as $\psi_{n} \in \mathcal{S}\left(\lambda^{n}\right)$ by definition, we know that each $\psi_{n}$ satisfies $\left\langle\psi_{n}, C_{i}^{n}\right\rangle \leq \lambda_{i}^{n}$ for every $i \in \mathcal{I}$. Given relation (7), we may pass to the limit $n \uparrow \infty$ to obtain the desired inequalities

$$
\left\langle\psi, C_{i}^{0}\right\rangle \leq \lambda_{i}^{0} \quad(i \in \mathcal{I})
$$

The first condition in (2) is stable with respect to weak convergence in $L^{1}(\mathbb{P} \otimes d E)$ and is thus inherited by $\psi$ from $\psi_{n}(n=1,2, \ldots)$.

Concerning the supporting property of $\psi$, note first that, from $\psi \geq b /(|\mathcal{I}|\langle B, E\rangle)$ it follows that

$$
\langle\psi, E\rangle \geq \frac{\langle b, E\rangle}{|\mathcal{I}|\langle B, E\rangle}>0 .
$$

Hence, in order to verify $\psi \in S\left(\lambda^{0}\right)$, it only remains to show that, under the price density $\psi$, every allocation $C \in \tilde{\mathcal{X}}_{+}^{\mathcal{I}}$ which is preferred to $C^{0}$ must have a higher aggregate price than $C^{0}$. By monotonicity of preferences, it suffices to consider a strictly preferred allocation $C$ in the sense that $V_{i}\left(C_{i}\right)>V_{i}\left(C_{i}^{0}\right)$ for every $i \in \mathcal{I}$. Due to Corollary $2.3 \mathrm{such}$ an allocation $C$ is also strictly preferred to $C^{n}$ when $n$ is large enough. As $\psi_{n} \in \mathcal{S}\left(\lambda^{n}\right)$ by assumption, each $C^{n}$ is supported by $\psi_{n}(n=1,2, \ldots)$. We thus obtain that, for $n$ sufficiently large,

$$
\left\langle\psi_{n}, \sum_{i} C_{i}\right\rangle \geq\left\langle\psi_{n}, E\right\rangle
$$

Letting $n \uparrow \infty$ in the preceding inequality gives us

$$
\left\langle\psi, \sum_{i} C_{i}\right\rangle \geq\langle\psi, E\rangle
$$

This shows that $\psi$ indeed supports $C^{0}$ and, therefore, completes the proof of assertion (ii). 
3. We still have to prove Bewley's claim (7). We follow his argument and note first that the claim already follows from the seemingly weaker assertion

$$
\limsup \left\langle\psi_{n}, C_{i}^{n}\right\rangle \leq\left\langle\psi, C_{i}^{0}\right\rangle \quad \text { for every } i \in \mathcal{I} \text {. }
$$

Indeed, the aggregation property of allocations and claim (8) imply

$$
\langle\psi, E\rangle \geq \limsup _{n} \sum_{i}\left\langle\psi_{n}, C_{i}^{n}\right\rangle=\limsup _{n}\left\langle\psi_{n}, E\right\rangle
$$

Due to the weak convergence $\psi_{n} \rightarrow \psi$, the last term is again equal to $\langle\psi, E\rangle$. Hence, we must have equality everywhere in (9) and claim (7) follows.

In order to establish (8), fix $i \in \mathcal{I}$ and set $C_{i}^{\epsilon} \triangleq C_{i}^{0}+\epsilon E$ for $\epsilon>0$. By monotonicity of preferences, we have $V_{i}\left(C_{i}^{\epsilon}\right)>V_{i}\left(C_{i}^{0}\right)$. Due to Corollary 2.3, we also have

$$
V_{i}\left(C_{i}^{\epsilon}\right)>V_{i}\left(C_{i}^{n}\right) \text { for large } n
$$

Since $\psi_{n}$ supports the allocation $C^{n}$, it follows that for such $n$

$$
\left\langle\psi_{n}, C_{i}^{\epsilon}+\sum_{j \neq i} C_{j}^{n}\right\rangle \geq\left\langle\psi_{n}, E\right\rangle
$$

or, equivalently,

$$
\left\langle\psi_{n}, C_{i}^{\epsilon}\right\rangle \geq\left\langle\psi_{n}, C_{i}^{n}\right\rangle
$$

Let $n$ tend to infinity and use the weak convergence $\psi_{n} \rightarrow \psi$ to conclude

$$
\left\langle\psi, C_{i}^{\epsilon}\right\rangle \geq \limsup _{n}\left\langle\psi_{n}, C_{i}^{n}\right\rangle
$$

Now, claim (8) follows from letting $\epsilon \rightarrow 0$ in the preceding estimate.

\section{$4 \quad$ Structure of Equilibrium Prices}

Having established existence of an equilibrium $\left(\left(C_{i}^{*}\right)_{i \in \mathcal{I}}, \psi^{*}\right)$, it is natural to ask, whether the induced equilibrium price functional $\left\langle\psi^{*},.\right\rangle$ is continuous on our consumption space $\left(\mathcal{X}_{+}, d_{\mathcal{X}_{+}}\right)$. Indeed, this is desirable from an economic point of view, since 'similar' consumption plans in $\mathcal{X}_{+}$should have 'similar' prices. We show in this section that under two additional assumptions, we indeed have this kind of continuity of the price functional.

Our first condition is

Assumption 3 The filtration $\mathbb{F}=\left(\mathcal{F}_{t}, 0 \leq t \leq T\right)$ is quasi left-continuous. 
This is an assumption on the way new information is revealed to the agents. It is satisfied if, e.g., the filtration $\mathbb{F}$ is generated by a Brownian motion or a Poisson process. Economically, an information flow corresponds to a quasi left-continuous filtration if 'information surprises' (in the sense of Hindy and Huang (1992)) occur only at times which cannot be predicted. An earthquake in New York (rather than San Francisco) is an example. The announcement of a policy change of the Federal reserve is an example for an information surprise which occurs at a time known in advance.

The second assumption allows us to use stochastic calculus:

Assumption 4 For every $C \in \mathcal{X}_{+}$, each utility gradient $\nabla V_{i}(C)(i \in \mathcal{I})$ is a bounded $(\mathbb{P}, \mathbb{F})$-semimartingale with a continuous compensator of bounded variation.

Clearly, this assumption is satisfied, e.g., if all agents have Hindy-Huang-Kreps preferences as described in Section 1.3.

Theorem 4.1 Under Assumptions 3 and 4, every equilibrium price functional

$$
C \mapsto\left\langle\psi^{*}, C\right\rangle \quad\left(C \in \mathcal{X}_{+}\right)
$$

is continuous on $\left(\mathcal{X}_{+}, d_{\mathcal{X}_{+}}\right)$.

\section{PROOF :}

1. As an equilibrium price process, $\psi^{*}$ clearly supports its associated equilibrium allocation $C^{*} \triangleq\left(C_{i}^{\lambda^{*}}\right)_{i \in \mathcal{I}}$. By Lemma 2.1 , it thus takes the form

$$
\psi^{*}=\max _{i}\left\{k_{i}^{*} \nabla V_{i}\left(C_{i}^{*}\right)\right\}
$$

for suitable constants $k_{i}^{*} \geq 0(i \in \mathcal{I})$.

Due to Assumption 4, each process $\phi_{i} \triangleq k_{i}^{*} \nabla V_{i}\left(C_{i}^{*}\right)(i \in \mathcal{I})$ allows a Doob-Meyer decomposition $\phi_{i}=M_{i}+A_{i}$ into a local martingale $M_{i}$ and a continuous compensator $A_{i}$ of bounded variation.

Moreover, defined as the pointwise maximum of the bounded semimartingales $\phi_{i}$, the process $\psi$ is a bounded semimartingale, too. Hence, it can be decomposed in the form $\psi=M+A$ where $M$ is a local martingale and $A$ is a predictable RCLL-process of bounded variation.

In particular, there is a localizing sequence of stopping times $T_{m}(m=1,2, \ldots)$ such that each of the stopped processes $M^{T_{m}}, M_{i}^{T_{m}}(i \in \mathcal{I})$ is a (uniformly integrable) martingale on $[0, T]$ and such that eventually $T_{m}(\omega)=T$ for $\mathbb{P}$-a.e. $\omega \in \Omega$.

2. We claim that $A$ almost surely has continuous paths. In order to prove this, it suffices to show that almost surely $A(S)=A(S-)$ for every predictable stopping time $S \leq T$ because both processes $(A(t),(0 \leq t \leq T)$ and $(A(t-), 0 \leq t \leq T)$ are predictable; cf., e.g., Rogers and Williams (1987), Lemma VI.19.2. 
Now, recall that, granted quasi-left continuity of the underlying filtration, every uniformly integrable martingale almost surely does not jump at predictable times; see, e.g., Theorem VI.18.1 in Rogers and Williams (1987).

We apply this observation first to the martingales $M_{i}^{T_{m}}$ and obtain that $\Delta M_{i}(S)=$ 0 on $\left\{S \leq T_{m}\right\}$ for every $i \in \mathcal{I}$. Since, in addition, each $A_{i}$ is continuous, this yields $\Delta \phi_{i}(S)=0$ on $\left\{S \leq T_{m}\right\}$ for every $i \in \mathcal{I}$. For $m \uparrow+\infty$, this entails $\Delta \phi_{i}(S)=0(i \in \mathcal{I})$ and, consequently, also $\Delta \psi(S)=0$ almost surely.

Applying the above observation to the stopped process $M^{T_{m}}$ shows that also $\Delta M(S)=0$ on $\left\{S \leq T_{m}\right\}$. Letting $m \uparrow \infty$ we obtain $\Delta M(S)=0$ almost surely. Together with $\Delta \psi(S)=0$, this implies $\Delta A(S)=0$ and we are done.

3. We are now in a position to prove the asserted continuity of the price functional $C \mapsto\left\langle\psi^{*}, C\right\rangle$. To this end, let $C^{n}(n=1,2, \ldots)$ converge to $C^{0}$ in the metric $d_{\mathcal{X}_{+}}$. Thus, we have $L^{1}(\mathbb{P})$-convergence of $C^{n}(T)$ to $C^{0}(T)$ and weak ${ }^{*}$-convergence in probability of the measures $d C^{n}$ to $d C^{0}$. By the usual subsequence argument, we may assume without loss of generality that both convergences hold true even almost surely.

Note that the local martingale $M$ is locally bounded since it is the difference of the bounded process $\psi$ and the continuous process $A$. Thus, we may assume that our localizing sequence $\left(T_{m}\right)$ is such that each $M^{T_{m}}(m=1,2, \ldots)$ is a bounded martingale.

For ease of notation, we put $\psi \triangleq \psi^{*}$. For every $m=1,2, \ldots$ we have

$$
\begin{aligned}
\left|\left\langle\psi, C^{n}\right\rangle-\left\langle\psi, C^{0}\right\rangle\right| \leq & \left|\mathbb{E} \int_{0}^{T}\left[\psi-\psi^{T_{m}}\right]\left(d C^{n}-d C^{0}\right)\right|+\left|\mathbb{E} \int_{0}^{T} \psi^{T_{m}}\left(d C^{n}-d C^{0}\right)\right| \\
\leq & \mathbb{E} \int_{T_{m}}^{T}\left|\psi-\psi\left(T_{m}\right)\right|\left(d C^{0}+d C^{n}\right) \\
& +\left|\mathbb{E} \int_{0}^{T} M^{T_{m}}\left(d C^{n}-d C^{0}\right)\right|+\left|\mathbb{E} \int_{0}^{T} A^{T_{m}}\left(d C^{n}-d C^{0}\right)\right| .
\end{aligned}
$$

Let us denote the preceding three summands by $I$, II, and III, respectively. For the first summand, we have

$$
I \leq\|\psi\|_{\infty} \mathbb{E}\left[\left(C^{n}(T)+C^{0}(T)\right) 1_{\left\{T_{m}<T\right\}}\right] .
$$

As $C^{n}(T) \rightarrow C^{0}(T)$ in $L^{1}(\mathbb{P})$ by assumption, dominated convergence yields

$$
\underset{n}{\limsup } I \leq 2\|\psi\|_{\infty} \mathbb{E}\left[C^{0}(T) 1_{\left\{T_{m}<T\right\}}\right] .
$$

Using the martingale property of $M^{T_{m}}$, we may rewrite the second summand in the form

$$
I I=\left|\mathbb{E}\left[M\left(T_{m}\right)\left(C^{n}(T)-C^{0}(T)\right)\right]\right| .
$$


Thus,

$$
\limsup _{n} I I \leq\left\|M\left(T_{m}\right)\right\|_{\infty} \limsup _{n} \mathbb{E}\left|C^{n}(T)-C^{0}(T)\right|=0 .
$$

Finally, note that, due to the continuity of $A$, we have

$$
\int_{0}^{T} A^{T_{m}} d C^{n} \rightarrow \int_{0}^{T} A^{T_{m}} d C^{0} \quad(n \uparrow \infty)
$$

almost surely. Moreover, we have

$$
\left|\int_{0}^{T} A^{T_{m}} d C^{n}\right| \leq\left\|\sup _{0 \leq t \leq T}\left|A^{T_{m}}(t)\right|\right\|_{\infty} C^{n}(T) .
$$

Once again we use the $L^{1}(\mathbb{P})$-convergence $C^{n}(T) \rightarrow C^{0}(T)$ and deduce that the right side of this estimate defines a uniformly integrable family of random variables parametrized by $n$. Hence, by Lebesgue's Theorem, we obtain that the convergence (10) holds true also in $L^{1}(\mathbb{P})$. For the third summand, this gives us

$$
\lim \sup I I I=0 .
$$

$n$

Summing up, we find that, for every $m=1,2, \ldots$,

$$
\begin{aligned}
\limsup _{n}\left|\left\langle\psi, C^{n}\right\rangle-\left\langle\psi, C^{0}\right\rangle\right| & \leq \underset{n}{\limsup I+\limsup _{n} I I+\limsup _{n} I I I} \\
& \leq 2\|\psi\|_{\infty} \mathbb{E}\left[C^{0}(T) 1_{\left\{T_{m}<T\right\}}\right]+0+0
\end{aligned}
$$

Letting $m \uparrow \infty$ in (11), we get by dominated convergence that indeed

$$
\underset{n}{\limsup }\left|\left\langle\psi, C^{n}\right\rangle-\left\langle\psi, C^{0}\right\rangle\right| \leq 2\|\psi\|_{\infty} \mathbb{E}\left[C^{0}(T) 1_{\bigcap_{m}\left\{T_{m}<T\right\}}\right]=0
$$

because $T_{m}(\omega)=T$ eventually for $\mathbb{P}$-a.e. $\omega \in \Omega$.

Properties of Equilibrium Price Processes We study now the properties of equilibrium price processes in more detail.

Being the weighted maximum of utility gradients, the equilibrium price process is a semimartingale if gradients are semimartingales. This is an important property because it provides an equilibrium foundation for the application of stochastic calculus in mathematical finance.

Going a step further, we see from the preceding proof that the bounded variation part of the equilibrium price process is continuous if gradients' bounded variation parts are continuous.

A fundamental question is whether this bounded variation part is even absolutely continuous because then an interest rate exists. The gradients of Hindy-Huang-Kreps 
utility functionals have such a nice representation. Hence, in a one consumer world, the Hindy-Huang-Kreps approach guarantees the existence of an interest rate. Hindy and Huang (1992) even suggest that also with heterogeneous agents, the equilibrium price process would have this nice property. However, this need not always be the case. From the Tanaka formula, it follows that the maximum of semimartingales whose finite variation part is absolutely continuous can be decomposed into a local martingale, an absolutely continuous part of bounded variation and a part which depends on local time of the gradients. In general, local times are not absolutely continuous. Hence, the equilibrium price process possibly does not belong to the dual suggested by Hindy and Huang (1992). Moreover, our characterization of supporting price functionals (Lemma 2.6) shows that, in general, there may be no equilibrium whose price process is contained in the Hindy-Huang dual.

Local time arises in the decomposition of the equilibrium price process whenever the identity of the agent whose gradient determines the price changes. Hence, whenever one agent stops consuming and another restarts, local time may appear. Such a phenomenon has already been remarked in the time-additive setting by Karatzas, Lehoczky, and Shreve (1991). For finance theory, this has the implication that the money market account contains a singular component. The detailed consequences for finance theory remain to be studied in future work.

\section{References}

BAnk, P., AND F. Riedel (1999): "Optimal Consumption Choice with Intertemporal Substitution under Uncertainty," Discussion paper 71, Sonderforschungsbereich 373, Humboldt University Berlin.

- (2000): "Optimal Consumption Choice with Intertemporal Substitution," mimeo, Humboldt University Berlin.

Bewley, T. (1969): "A Theorem on the Existence of Competitive Equilibria in a Market with a Finite Number of Agents and whose Commodity Space is $L^{\infty}$," Discussion paper, CORE, Université de Louvain.

Duffie, D., And L. Epstein (1992): "Asset Pricing with Stochastic Differential Utility," Review of Economic Studies, 5, 411-436.

Duffie, D., and C. Skiadas (1994): "Continuous-Time Security Pricing, a Utility Gradient Approach," Journal of Mathematical Economics, 23, 107-131.

Hindy, A., And C.-F. HuAng (1992): "Intertemporal Preferences for Uncertain Consumption: a Continuous-Time Approach," Econometrica, 60, 781-801.

Hindy, A., C.-F. HuAng, And D. Kreps (1992): "On Intertemporal Preferences in Continuous Time - the Case of Certainty," Journal of Mathematical Economics, 21, 401-440. 
Hindy, A., C.-F. Huang, and S. Zhu (1997): "Optimal Consumption and Portfolio Rules with Durability and Habit Formation," Journal of Economic Dynamics and Control, 21, 525-550.

Kabanov, Y. (1999): "Hedging and Liquidation under Transaction Costs in Currency Markets," Finance and Stochastics, 2, 237-248.

Karatzas, I., J. P. Lehoczky, and S. E. Shreve (1991): "Equilibrium Models with Singular Asset Prices," Mathematical Finance, 1, 11-29.

Komlós, J. (1967): "A Generalization of a Problem of Steinhaus," Acta Math. Acad. Sci. Hung, 18, 217-229.

Mas-Colell, A., and S. Richard (1991): "A New Approach to the Existence of Equilibrium in Vector Lattices," Journal of Economic Theory, 53, 1-11.

Mas-Colell, A., And W. Zame (1991): "Equilibrium Theory in Infinite Dimensional Spaces," in Handbook of Mathematical Economics, ed. by W. Hildenbrand, and H. Sonnenschein, vol. IV, pp. 1835-1898. North-Holland.

Rogers, C., And D. Williams (1987): Diffusions, Markov Processes, and Martingales, vol. 2. Wiley.

PETER BANK

Institut für Mathematik

Humboldt-Universität zu Berlin

Unter den Linden 6

D-10099 Berlin, Germany

E-mail: pbank@mathematik.hu-berlin.de E-mail: riedel@odin.wiwi.hu-berlin.de
FRANK RIEDEL

Institut für Wirtschaftstheorie I

Humboldt-Universität zu Berlin

Spandauer Straße 1

D-10178 Berlin, Germany 